\title{
Fibrinogen interaction with complement C3: a potential therapeutic target to reduce thrombosis risk
}

Haematologica 2021

Volume 106(6):1616-1623

\section{Correspondence:}

RA AJJAN

r.ajjan@leeds.ac.uk

Received: October 1, 2019.

Accepted: April 29, 2020.

Pre-published: April 30, 2020.

https://doi.org/10.3324/haematol.2019.239558

(C)2021 Ferrata Storti Foundation

Material published in Haematologica is covered by copyright. All rights are reserved to the Ferrata Storti Foundation. Use of published material is allowed under the following terms and conditions:

https://creativecommons.org/licenses/by-nc/4.0/legalcode. Copies of published material are allowed for personal or internal use. Sharing published material for non-commercial purposes is subject to the following conditions:

https://creativecommons.org/licenses/by-nc/4.0/legalcode, sect. 3. Reproducing and sharing published material for commercial purposes is not allowed without permission in writing from the publisher.

\section{Rhodri J. King, ${ }^{1 *}$ Katharina Schuett, ${ }^{2 *}$ Christian Tiede, ${ }^{3}$ Vera Jankowski, ${ }^{4}$ Vicky John, ${ }^{1}$ Abhi Trehan, ${ }^{1}$ Katie Simmons, ${ }^{5}$ Sreenivasan Ponnambalam, ${ }^{6}$ Robert F. Storey, ${ }^{7}$ Colin W.G. Fishwick, ${ }^{5}$ Michael J. McPherson, ${ }^{3}$ Darren C. Tomlinson ${ }^{3}$ and Ramzi A. Ajjan ${ }^{1}$}

${ }^{1}$ Leeds Institute for Cardiovascular and Metabolic Medicine, Leeds University, Leeds, UK; ' ${ }^{2}$ Department of Internal Medicine I, University Hospital Aachen, Aachen, Germany, ${ }^{3}$ Bioscreening Technology Group in the School of Molecular and Cellular Biology, University of Leeds, Leeds, UK, ${ }^{4}$ Institute for Molecular and Cardiovascular Research, Aachen University, Aachen, Germany, ${ }^{5}$ School of Chemistry, University of Leeds, Leeds, UK, ${ }^{6}$ School of Molecular \& Cellular Biology, University of Leeds, Leeds, UK and ${ }^{7}$ School of Medicine, University of Sheffield, Sheffield, UK

${ }^{*} R J K$ and $K S$ contributed equally as co-first authors.

\section{ABSTRACT}

omplement C3 binds fibrinogen and compromises fibrin clot lysis, thereby enhancing the risk of thrombosis. We investigated the role of the fibrinogen-C3 interaction as a novel therapeutic target to reduce thrombosis risk by analyzing: (i) consistency in the fibrinolytic properties of C3; (ii) binding sites between fibrinogen and C3; and (iii) modulation of fibrin clot lysis by manipulating fibrinogen-C3 interactions. Purified fibrinogen and C3 from the same individuals ( $n=24)$ were used to assess inter-individual variability in the anti-fibrinolytic effects of C3. Microarray screening and molecular modeling evaluated C3 and fibrinogen interaction sites. Novel synthetic conformational proteins, termed affimers, were used to modulate the C3-fibrinogen interaction and fibrinolysis. C3 purified from patients with type 1 diabetes showed enhanced prolongation of fibrinolysis compared with healthy control protein $(195 \pm 105$ and $522 \pm 166 \mathrm{~s}$, respectively; $P=0.04)$, with consistent effects but a wider range (5-51\% and 5-18\% lysis prolongation, respectively). Peptide microarray screening identified two potential C3-fibrinogen interaction sites within the fibrinogen $\beta$ chain (residues $424-433$ and 435-445). One fibrinogen-binding affimer that was isolated displayed sequence identity with C3 in an exposed area of the protein. This affimer abolished C3induced prolongation of fibrinolysis $(728 \pm 25.1 \mathrm{~s}$ to $632 \pm 23.7 \mathrm{~s} ; P=0.005)$ and showed binding to fibrinogen in the same region that is involved in C3-fibrinogen interactions. Moreover, it shortened plasma clot lysis of patients with diabetes, cardiovascular disease or controls by $7-11 \%$. C3 binds fibrinogen $\beta$-chain and disruption of the fibrinogen-C3 interaction using affimer proteins enhances fibrinolysis, which represents a potential novel tool to reduce thrombosis in high-risk individuals.

\section{Introduction}

Hypofibrinolysis is associated with increased risk of atherothrombotic events. ${ }^{1.3}$ Although most studies have only shown an association between hypofibrinolysis and cardiovascular disease, a more recent longitudinal study in a large population of patients with acute coronary syndrome demonstrated that prolonged fibrin clot lysis is an independent predictor of cardiovascular mortality. ${ }^{4}$ Therefore, it was proposed that reducing residual thrombosis risk in patients with coronary artery disease requires targeting the fibrinolytic system in addition to administering antiplatelet therapies. Indeed the combination of anticoagulant and antiplatelet therapies reduces vascular thrombotic events but at the expense of increased risk of bleeding. ${ }^{5}$ Therefore, a more targeted approach is required which improves hypofibrinolysis 
and reduces the risk of vascular thrombosis without increasing bleeding events.

Complement C3, which is incorporated into fibrin clots, has been shown to modulate fibrin clot lysis, ${ }^{6}$ an effect that may be exaggerated in patients with higher vascular risk. ${ }^{7}$ C3 plasma levels have shown correlations with ex-vivo plasma clot lysis in individuals with diabetes as well as in healthy controls. ${ }^{7,8}$ Moreover, plasma levels of this protein were independent predictors of resistance to fibrinolysis in 875 high-vascular-risk patients with type 2 diabetes. ${ }^{9}$ These data suggest that C3 represents a credible therapeutic target to shorten fibrin clot lysis and ultimately reduce thrombosis risk. However, these functional studies were conducted using pooled C3 and studies assessing consistency of the response in different individuals, crucial to establish the therapeutic potential of C3, are lacking.

We have recently demonstrated that fibrin clot lysis can be modulated, leading to stabilization of the clot, by using small conformational proteins, termed affimers. ${ }^{10,11}$ Affimer technology was able to correct abnormal lysis of clots made from plasma samples of individuals with hemophilia, indicating a potential therapeutic role for these proteins. However, it remains unknown whether affimers can be used to enhance clot lysis, particularly in individuals at high vascular risk.

The aim of this work was to establish the role of C3 as a therapeutic target for enhancing fibrinolysis and reducing thrombosis risk. Therefore, we studied: (i) consistency in the fibrinolytic properties of C3 in healthy controls and patients with high vascular risk; (ii) potential binding sites between fibrinogen and C3; and (iii) modulation of fibrin clot lysis by targeted interference of fibrinogen-C3 interactions.

\section{Methods}

Only a brief description is provided here; full details can be found in the Online Supplement.

\section{Recruitment of patients}

We recruited 12 patients with type 1 diabetes (T1DM) and 12 age- and sex-matched controls to purify fibrinogen and C3. We additionally tested 24 stored plasma samples from individuals with high vascular risk. Ethical approval for the study was provided by the Leeds East, National Research Ethics Service committee and informed consent was obtained from participants in accordance with the Declaration of Helsinki.

\section{Protein purification and turbidimetric analysis}

Fibrinogen and complement C3 were purified as described elsewhere ${ }^{7}$ and clots were formed from purified fibrinogen in the presence or absence of corresponding C3 from the same individual. Time from full clot formation to $50 \%$ lysis was calculated and is presented as clot lysis time throughout this work.

\section{Peptide microarray}

A peptide microarray chip containing overlapping 15 amino acid peptides of the fibrinogen molecule (PEPperPRINT, Germany) was synthesized to screen against pooled C3 $(0.8$ to $8 \mu \mathrm{g} / \mathrm{mL})$ purified from six healthy individuals.

\section{Non-antibody synthetic proteins (affimers)}

Affimers are small proteins composed of a scaffold protein that constrains two variable conformational nine amino acid loops. ${ }^{11,12}$

\section{Panning and protein production}

A phage display library of affimer proteins, comprising $1.3 \times 10^{10}$ random variable clones, was screened against human fibrinogen. In order to select specific affimers capable of targeting fibrinogenC3 interactions, competitive elution with C3 for 20 min was applied in the fourth panning.

\section{Affimers and fibrinolysis}

Fibrinogen was incubated with affimer $\mathrm{A} 6$ at room temperature for 30 min after which C3 and factor XIII were added and clot formation/lysis was triggered as described previously. ${ }^{13}$

Plasma samples were mixed with increasing concentrations of affimer A6 or control scaffold and then turbidimetric experiments were performed, as described elsewhere. ${ }^{13}$

\section{Molecular modeling}

In order to identify potential sites of ligand binding on $\mathrm{C} 3$, the molecular modeling software Autoligand was employed to scan the whole protein. ${ }^{14}$ Molecular modeling was used to predict binding sites of any peptide sequences identified from the microarray screening and affimer work using the webserver Pepsite $2^{\circledR}$ (http://pepsite2.russelllab.org). Images were viewed and produced using Pymol (The PyMOL Molecular Graphics System, Version 2.0 Schrödinger, LLC). ${ }^{15}$

\section{Mass spectrometry}

Purified C3 from six subjects with type 1 diabetes mellitus and six healthy controls was digested by trypsin and analyzed by matrix-assisted laser desorption/ionization -time-of-flight mass spectrometry (MALDI-TOF/MS) as described elsewhere. ${ }^{16}$ To compare potential differences between in vivo and in vitro C3 glycation, we undertook in vitro glycation of the protein as previously described ${ }^{17}$ and post-translational modifications were assessed as above.

\section{Statistical analysis}

Between-group comparisons of normally and non-normally distributed variables were carried out by independent Student $t$ and Mann-Whitney U tests, respectively. Pearson and Spearman coefficients were applied to assess correlations between normally and non-normally distributed variables, respectively. Based on previous data ${ }^{7}$ and assuming a common standard deviation of the variable studied of 225 seconds (s) we calculated that the number of samples analyzed ( $n=24$ in total) would be enough to detect a difference of $270 \mathrm{~s}$ in C3-induced prolongation of clot lysis time (power $80 \%$ at $P<0.05$ ).

\section{Results}

\section{Patients' characteristics}

Twelve patients with type 1 diabetes and 12 healthy controls were recruited. Their characteristics are shown in Table 1 . The patients with diabetes were on no medications other than subcutaneous insulin injections and had no clinically significant microvascular or macrovascular complications of diabetes. The healthy controls were also not taking any prescribed medication.

\section{Effect of C3 on lysis time}

One control sample did not lyse within the allotted time and was, therefore, excluded from further analysis. The mean lysis time of all clots made from fibrinogen in the absence and presence of corresponding C 3 was $2691 \pm 105$ and $3057 \pm 172 \mathrm{~s}(P<0.01)$. The mean lysis time of clots 
made from purified fibrinogen of healthy controls and patients with type 1 diabetes was $2510 \pm 132 \mathrm{~s}$ and $2857 \pm 151 \mathrm{~s}$, respectively $(P=0.07)$. Following the addition of $\mathrm{C} 3$, there was an increase in lysis time in both groups to $2705 \pm 180 \mathrm{~s}$ and $3379 \pm 259 \mathrm{~s}$, respectively, with individual data provided in Figure 1A. The degree of C3-induced prolongation in lysis time was greater in the diabetes samples $(179 \pm 101$ and $522 \pm 166 \mathrm{~s} ; P=0.04)$ (Figure $1 \mathrm{~B}$ and $\mathrm{C}$ ), and showed a broader range of 144-1476 s (5-51\%) among the patients with diabetes, compared with the 108-480 s $(5-18 \%)$ in controls. These data show that C3 consistently prolongs clot lysis time in both healthy controls and patients with diabetes, although there was wider interindividual variability in the degree of prolongation in the group with diabetes.

\section{C3-fibrinogen interaction sites}

Our data have so far shown that C3 represents a credible target to modulate fibrinolysis and we therefore investigated fibrinogen-C3 binding sites. Using peptide microarray screening of the whole fibrinogen molecule, we identified complex spot patterns, based on peptides with the consensus motifs $\mathrm{A}^{136}$ VSOTSSSSFOYMYL ${ }^{150}$ (peptide A), ${ }^{423}$ OCSKEDGGGWWY ${ }^{434}$ (peptide B) and ${ }^{434} Y_{N R C H A A N P N G R Y Y}{ }^{447}$ (peptide C), which are all located within the $\beta$ chain of fibrinogen (Uniprot ID
Table 1. Clinical variables of controls and patients with type 1 diabetes. Data are presented as mean \pm standard error of mean.

\begin{tabular}{|c|c|c|c|}
\hline Variable & $\begin{array}{c}\text { Type } 1 \text { diabetes } \\
(n=12)\end{array}$ & $\begin{array}{c}\text { Controls } \\
(n=12)\end{array}$ & $P$ value \\
\hline Age (years) & $25.1 \pm 1.7$ & $27.9 \pm 1.3$ & 0.2 \\
\hline $\operatorname{Sex}(M / F, n)$ & $7 / 5$ & $9 / 3$ & \\
\hline Duration of diabetes (months) & $108 \pm 68$ & - & \\
\hline Current smokers (n) & 2 & 0 & \\
\hline Systolic BP (mmHg) & $119 \pm 4.6$ & $121 \pm 3.8$ & 0.73 \\
\hline Diastolic BP (mmHg) & $76 \pm 4$ & $76 \pm 2.5$ & 0.85 \\
\hline BMI $\left(\mathrm{kg} / \mathrm{m}^{2}\right)$ & $25.4 \pm 0.96$ & $23.5 \pm 0.6$ & 0.12 \\
\hline $\mathrm{C} 3(\mathrm{mg} / \mathrm{mL})$ & $1.34 \pm 0.06$ & $1.39 \pm 0.07$ & 0.54 \\
\hline Fibrinogen $(\mathrm{mg} / \mathrm{mL})$ & $2.0( \pm 0.32)$ & $2.2( \pm 0.27)$ & 0.2 \\
\hline Plasma glucose (mmol/L) & $12.8 \pm 1$ & $4.4 \pm 0.2$ & $<0.0001$ \\
\hline Total cholesterol (mmol/L) & $4.8 \pm 0.35$ & $4.8 \pm 0.32$ & 0.97 \\
\hline Triglycerides (mmol/L) & $1.3 \pm 0.15$ & $1.1 \pm 0.11$ & 0.16 \\
\hline TSH (mU/L) & $2.5 \pm 0.32$ & $2.1 \pm 0.18$ & 0.33 \\
\hline Free T4 $(\mathrm{pmol} / \mathrm{L})$ & $14.3 \pm 0.39$ & $15 \pm 0.45$ & 0.24 \\
\hline Creatinine $(\mu \mathrm{mol} / \mathrm{L})$ & $76.8 \pm 4.6$ & $81 \pm 4.2$ & 0.52 \\
\hline Plasma clot lysis (seconds) & $427.5 \pm 14.6$ & $425 \pm 7.9$ & 0.88 \\
\hline
\end{tabular}

M: male, F: female; BP: blood pressure; BMI: body mass index; C3: complement C3; TSH: thyroidstimulating hormone, T4: thyroxine.
A

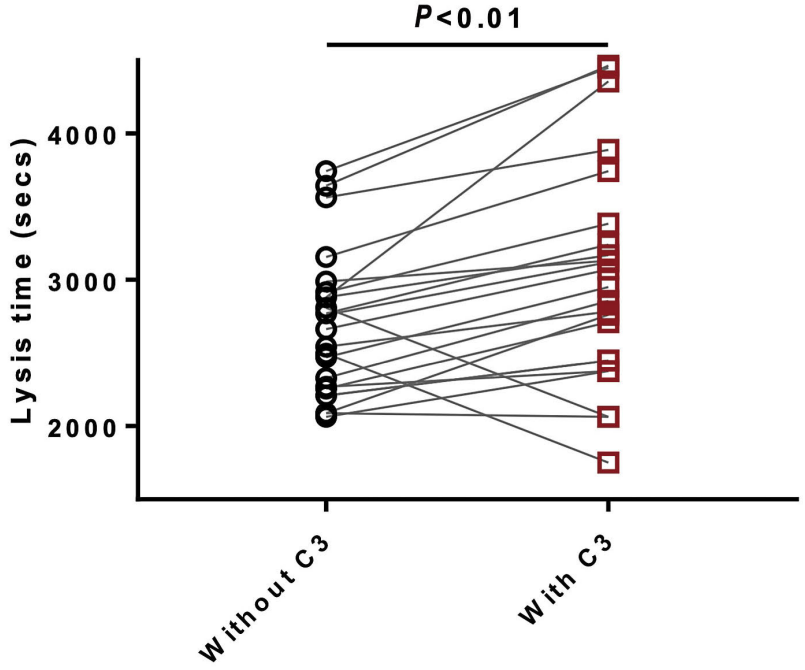

B

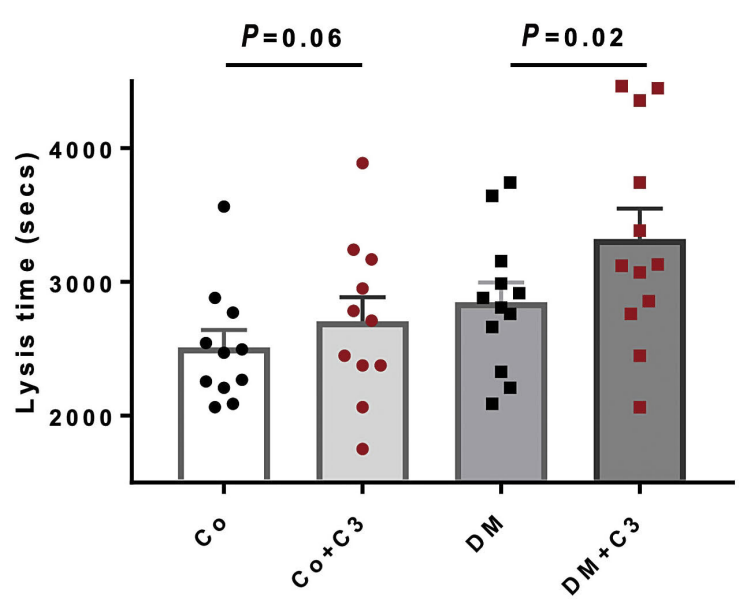

C

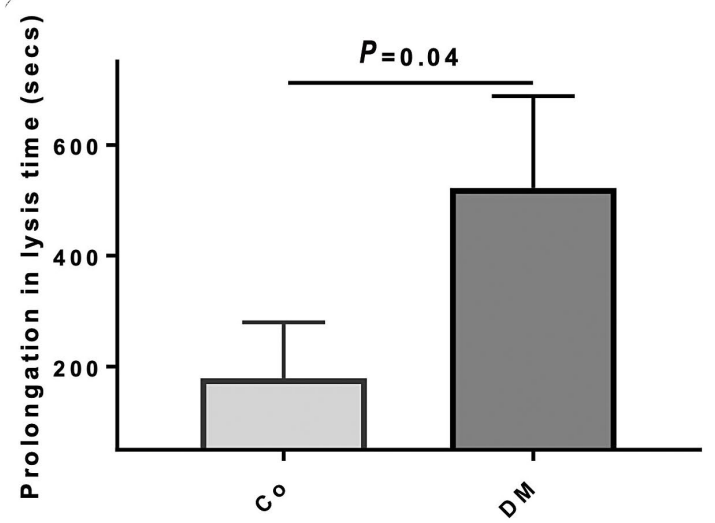

Figure 1. C3 modulation of clot lysis in individuals with diabetes and healthy controls. Fibrinogen and $\mathrm{C} 3$ were purified from matched samples of 12 patients with diabetes and 12 healthy controls. Turbidimetric experiments were conducted on individual fibrinogen samples after the addition of thrombin, calcium, tissue plasminogen activator and plasminogen, in the presence and absence of C3. (A) Clot lysis time was increased following the addition of C3 when all samples $(n=24)$ were analyzed together. (B) Lysis time of individual samples from healthy controls and patients with diabetes before and after the addition of $\mathrm{C} 3$ ( $n=12$ in each group), (C) Prolongation in lysis time by $\mathrm{C} 3$ in samples from healthy controls and patients with diabetes ( $n=12$ in each group). Data are presented as mean \pm standard error of mean. Co: controls; DM: patients with diabetes mellitus. 
P02675). Two of these peptides (B and C) were in close proximity in the $\mathrm{N}$ terminus of the $\beta$-chain and shared one amino acid. One peptide was closer to the C-terminal region of the $\beta$ chain of fibrinogen, as shown in Figure 2. These data indicate that the $\beta$ chain of fibrinogen contains key binding sites for C3.

\section{Use of affimers for modulation of C3-induced prolongation of fibrinolysis \\ Isolation of affimer $A 6$}

Sixteen fibrinogen-binding affimers, which competed with C3 for fibrinogen binding, were sequenced and revealed eight distinct sequences. Each affimer is composed of a scaffold protein and two nine amino acid loops. We identified one affimer (A6) which showed sequence identity in one of its loops with residues Ser 38-His 47 of C3 (Uniprot ID P01024) (Figure 3A). This site resides in an exposed area of C3 with the potential to interact with other plasma proteins, as predicted by molecular modeling (Figure $3 \mathrm{~B}$ ).

\section{Effects of affimer A6 on fibrinolysis}

Using pooled fibrinogen from healthy controls, we investigated changes in lysis time after the addition of A6, C3 or a combination of the two. A6 did not have a significant effect on clot lysis whereas C3 prolonged clot lysis, an effect that was completely abolished by the addition of A6 (Figure 4A). Affimer A6 had no effect on lysis time when C3 was substituted by plasmin inhibitor, indicating a C3-specific effect (Figure 4A).

Given the results of the purified protein experiments, we next analyzed plasma samples. A dose-response curve, using pooled healthy control plasma samples, verified that a 1:1 molar concentration of fibrinogen:affimer A6 is optimal for modulation of clot lysis (Figure 4B). Using this molar ratio, affimer A6 reduced the lysis time of clots made from pooled healthy control plasma from $593 \pm 17$ s to $542 \pm 10 \mathrm{~s}(7 \%, P<0.05)$ with a decrease from $618 \pm 13 \mathrm{~s}$ to $548 \pm 15 \mathrm{~s}$ for clots made from plasma from patients with diabetes $(11 \%, P<0.05)$ (Figure $4 \mathrm{C})$. We also tested the effect of affimer A6 in individual samples and not only pooled plasma. Affimer A6 reduced plasma clot lysis in 24 patients with high vascular risk (11 with coronary artery disease and 13 with type 2 diabetes) from $1237 \pm 150$ s to $1120 \pm 129$ s $(10 \%, P<0.05)$ (Figure 4D).

\section{Binding sites of affimer A6 on fibrinogen}

Following the results of the microarray screening of C3 and fibrinogen, the sequences for the two random loops of A6 were individually entered into the Pepsite 2 webserver to predict where $\mathrm{A} 6$ might be binding to the $\beta$-chain of fibrinogen (PDB code $3 \mathrm{GHG} ;{ }^{18}$ ). The strongest binding

$A^{-}$

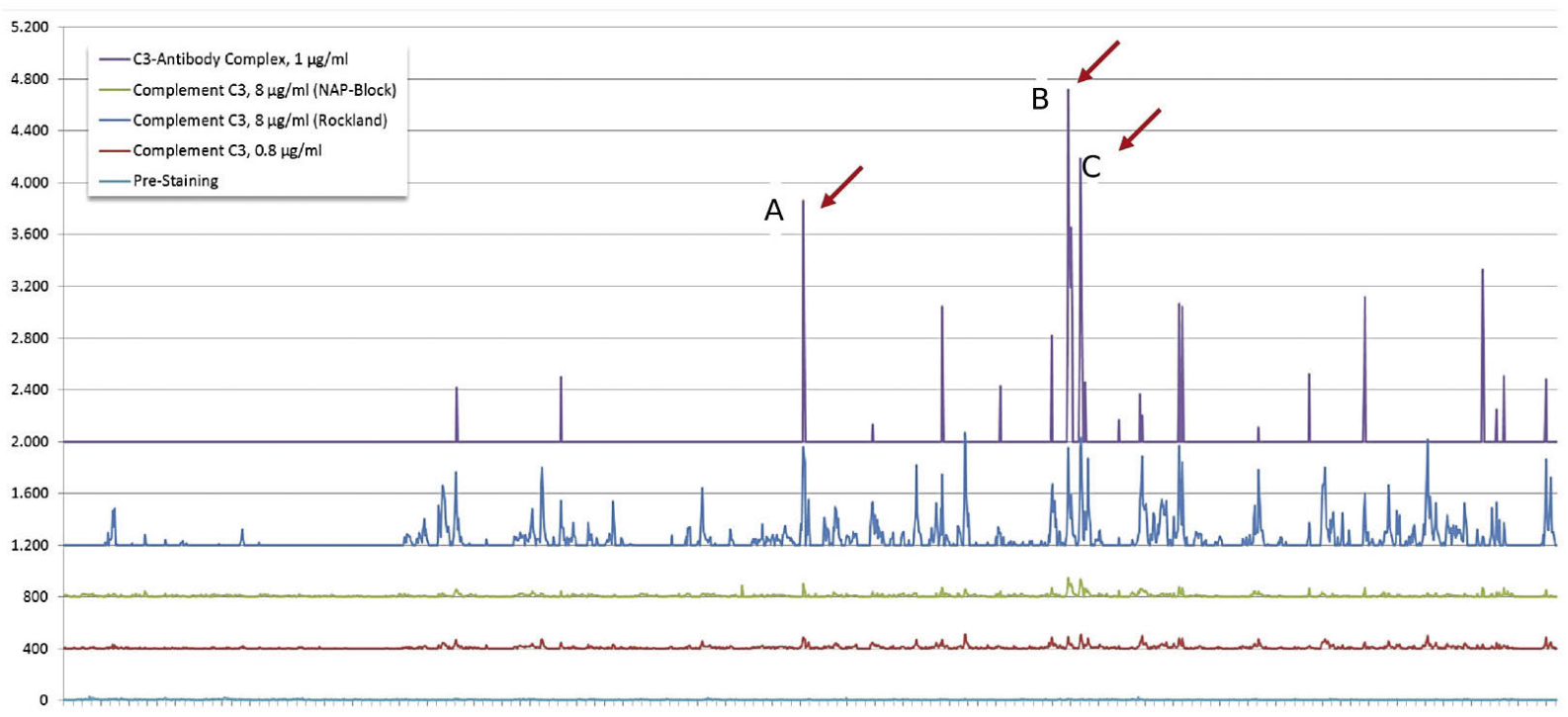

B

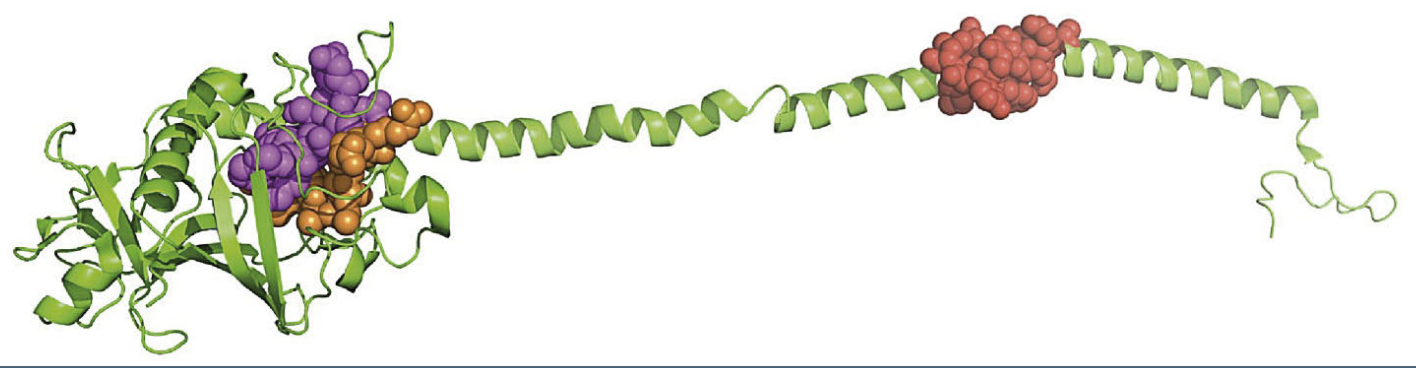

Figure 2. Fibrinogen peptide binding to $\mathrm{C} 3$ and location within the fibrinogen molecule. (A) Intensity plot of fibrinogen peptide binding to $\mathrm{C} 3$ showing mainly three binding sites (visualized as 3 distinct intensity peaks, red arrows), all located on the fibrinogen $\beta$ chain (A, B and C). (B) The peptide sequences identified by the microarray screening are illustrated within the crystal structure of the $\beta$ chain of fibrinogen. The red spheres indicate peptide $A\left({ }^{136} A V S Q T S S S S F Q Y M Y L{ }^{150}\right)$, pink spheres indicate peptide B $\left({ }^{423} \mathrm{QCSKEDGGGWWY}{ }^{434}\right)$ and orange spheres indicate peptide C $\left({ }^{434}\right.$ YNRCHAANPNGR $\left.{ }^{445}\right)$. 
prediction for the two loops on the $\beta$ chain was adjacent to the peptide sequences (peptides B and C) identified by the microarray screening (Figure 4E).

\section{Mechanistic insight into potential enhancement of fibrinolysis prolongation by $\mathrm{C} 3$ from patients with diabetes}

We have previously shown that pooled C3 purified from patients with diabetes may have enhanced anti-fibrinolytic effects compared with the protein from healthy controls. The current work, using C3 purified from individual plasma samples, demonstrates that C3 from patients with diabetes has enhanced anti-fibrinolytic activity compared with control protein, at least in some samples. We therefore investigated whether increased protein glycation may be one of the mechanisms involved in the enhanced anti-fibrinolytic effects of C3. Mass spectrometry identified similar glycation of amino acids serine, threonine, arginine, and asparagine in C3 samples from controls and patients with diabetes. However, additional lysine residues were noted to be glycated in all six diabetes samples but not in the control group, affecting six separate residues, with a mean of $3 \pm 0.9$ modifications in each sample. Details of the glycated lysine residues and positions within C3 are illustrated in Figure 5A and B. A characteristic mass-spectrum of a fragment of C3 analyzed by tandem mass spectrometry, with the molecular weight of 1,206 Daltons after tryptic digestion, is shown in Figure $5 \mathrm{C}$ together with the protein glycation score. We verified the molecular mass of 1,206 as a characteristic post-translational glycation modification of the fragment with the molecular weight of 882 of C3. This molecular mass $1,206 \mathrm{~m} / \mathrm{z}$ shows glycation of lysine, highlighted by an asterisk in the amino acid sequence $\beta K^{*}$ GPLLNK ${ }^{* 1209}$. Although the additional glycation of C3 in patients with diabetes was associated with longer clot lysis time, the Spearman coefficient did not demonstrate a significant correlation between number of glycated lysine residues within C3 and ex vivo plasma clot lysis time (Spearman $\mathrm{r}=0.19, P=0.73)$.

\section{Discussion}

There are a number of novel findings in this work that can be summarized as follows: (i) C3 purified from individual patients consistently prolongs clot lysis; (ii) the $\beta$ chain of fibrinogen in the area of Cys424-Arg445 represents one region of interaction with complement C3; and (iii) affimer proteins provide a tool for targeted modula-

A

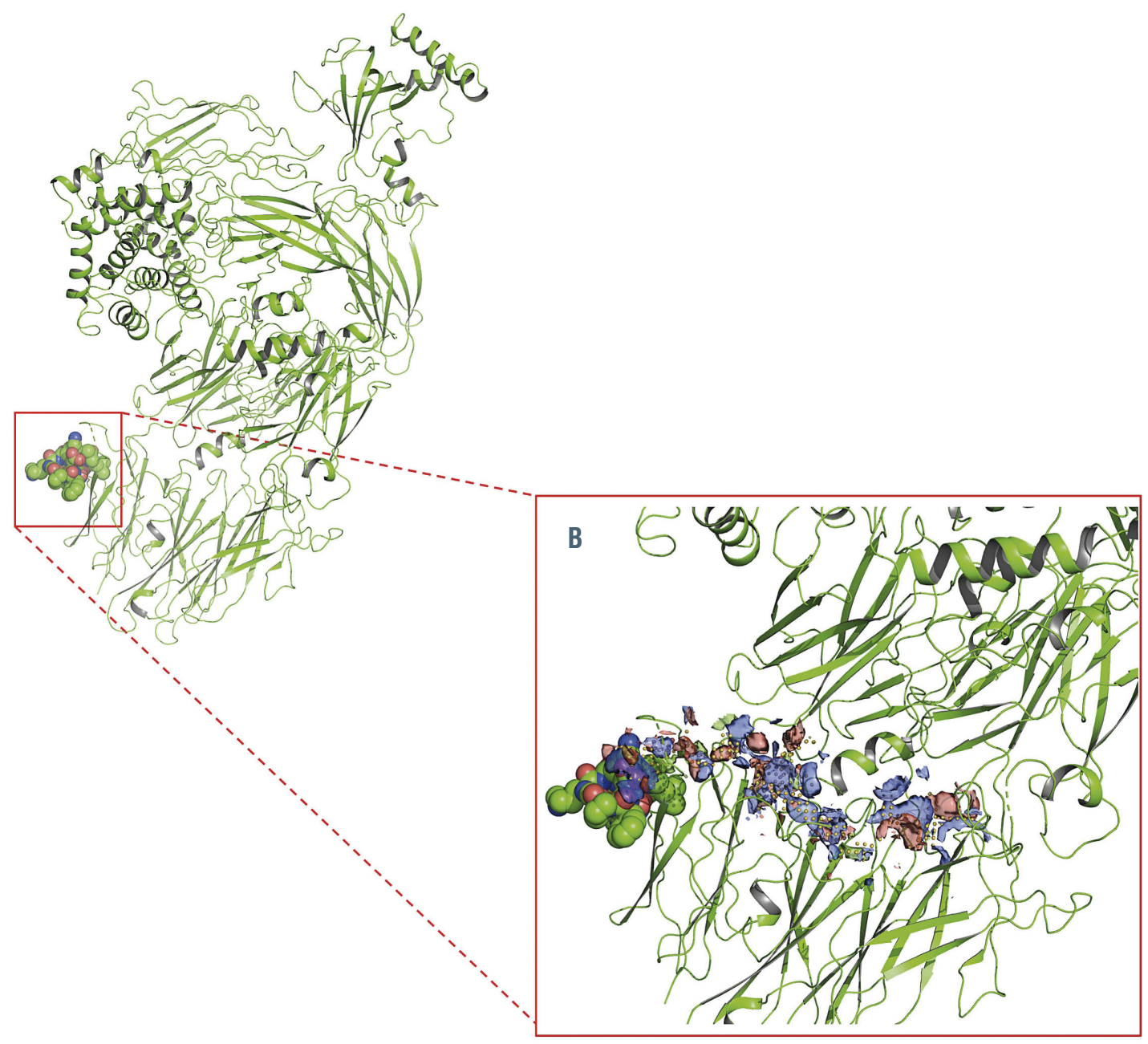

Figure 3. Affimer A6 sequence identity with $\mathrm{C} 3$ and potential protein-protein interaction sites on C3. (A) Loop 2 of affimer A6 showing sequence identity with the area represented by red spheres on the $\mathrm{C} 3$ molecule. (B) Enlargement of the area of potential protein-protein interaction sites on complement C3 (labeled in different colors) identified as the best scoring site using the SiteMap module of Glide (Schrodinger Inc). 
tion of fibrin clot lysis by interfering with fibrinogen-C3 interactions.

While C3 protein purified from individual plasma samples consistently prolonged clot lysis, an inter-individual variability in the response was noted, which may be related to the degree of incorporation of C3 into the clot. ${ }^{19}$ This was particularly pronounced in C3 purified from patients with diabetes, which may be related to alternative posttranslational modifications in the protein. We identified six lysine residues that were glycated in samples from patients with type 1 diabetes, but not in samples from controls. The variability in glycation sites comparing individuals with type 1 diabetes suggests that the ability of lysine residues on C3 to undergo glycation has a large inter-individual variability, which may modulate the antifibrinolytic effects of the protein. Interestingly, in vitro glycation of C3 showed largely similar patterns, favoring glycation of certain lysine residues, suggesting this consis-
A

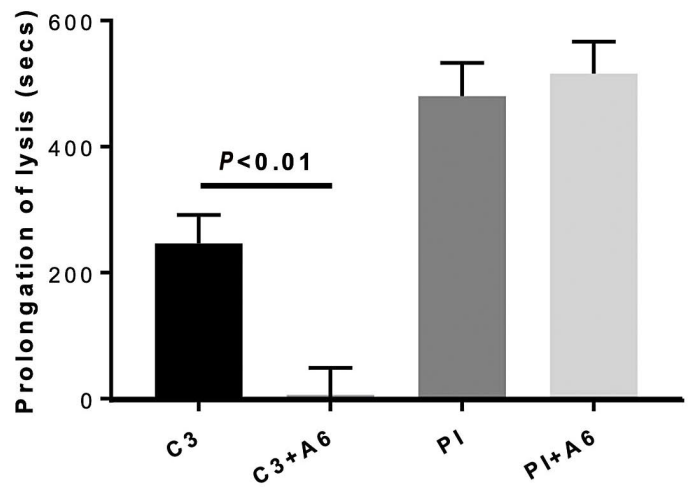

A 6 and C 3 -induced lysis prolongation on lysis (purified)

C

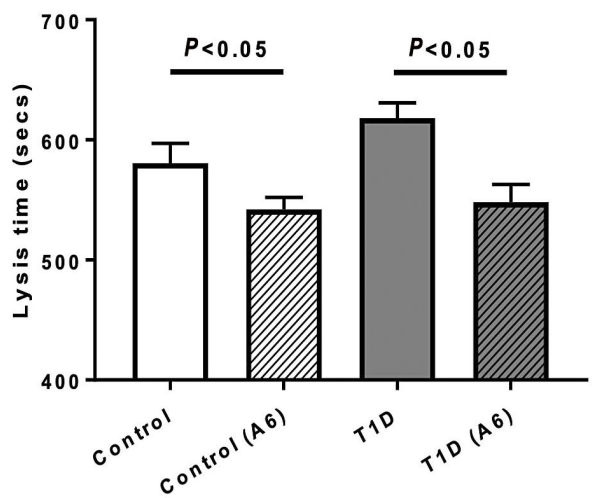

D

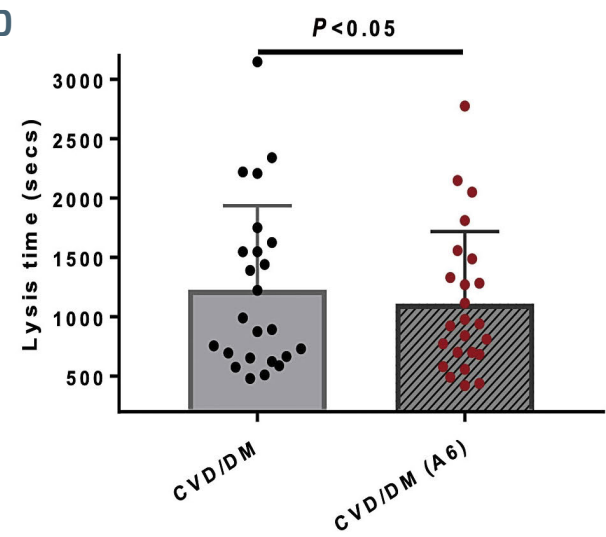

Figure 4. Modulation of C3-induced prolongation of fibrinolysis by affime A6 and pontential interaction sites on fibrinogen. (A) Pre-incubation of fibrinogen with affimer A6 completely abolished complement C3-mediated prolongation of lysis with no effect observed on prolongation of lysis by plasmin inhibitor indicating a C3-specific effect. (B) Dose-response curve using different mola ratios of affimer A6:fibrinogen (or scaffold-only control protein), conducted on pooled plasma samples from 12 individuals. (C) Effects of affime A6 on plasma clot lysis using pooled healthy control plasma (control, $n=12$ ) or plasma from patients with type 1 diabetes (patients, $n=12$ ). Results represent the mean \pm standard error of mean of three independ ent experiments each performed in duplicate, unpaired $t$-test. (D) The effect of affimer $A 6$ on plasma clot lysis in 24 patients with high vascular risk (11 with coronary artery disease and 13 with type 2 diabetes). (E) The crystal structure of fibrinogen with predicted binding sites of loop 1 (red) and loop 2 (blue) of affimer A6 within the chain (shown in green), lying in close proximity to peptide motifs $B$ and $C$ identified from microarray screening (colored spheres). C3: complement C3; A6: affimer A6 PI: plasmin inhibitor; T1D: type 1 diabetes; CVD: cardiovascular disease; DM: diabetes mellitus. 
tency is related to a steric effect. Previous work has shown that glycation of proteins involved in coagulation and lysis alters clot structure and/or the efficiency of fibrinolysis. For example, fibrinogen glycation alters fibrin network characteristics and the degree of protein glycation correlates with glycemic control measured as HbA1c. ${ }^{20,21}$ Proteins in the fibrinolytic system are also affected, as plasminogen glycation in diabetes compromises conversion to plasmin and modulates enzyme activity. ${ }^{22}$ There was no clear correlation between the number of lysine residues glycated and the antifibrinolytic effects of C3 but this may be due to the small number of samples analyzed or it may simply indicate that some lysine residues are more important than others and extensive glycation of multiple residues is not required to observe an effect. Overall, however, our data suggest that glycation of C3 increases its antifibrinolytic effect, although it remains unclear which lysine residues are important for the observed effect.

Our binding studies indicate that three areas on the $\beta$ chain of fibrinogen play a role in C3-fibrinogen interactions. In particular, two of these areas, located in the $\mathrm{N}$-terminus, were in close proximity and separated by a single amino acid. We should acknowledge that the microarray technique only identifies linear interactions and it is possible that additional conformational interactions take place between the two proteins. However, we should not underestimate the importance of linear binding between proteins with previous work demonstrating the importance of such interactions between fibrinogen and factor XIII. ${ }^{23}$

A key finding of our work is a proof of concept for a novel methodology to modulate fibrin clot lysis, and hence thrombosis risk, in individuals with diabetes. We describe a new technique that identified a small binding protein with two variable loops (affimer A6), with one of the loops sharing sequence identity with an exposed portion of C3, likely to be involved in protein-protein interactions. Interestingly, affimer A6 was able to abolish C3induced prolongation of lysis with high specificity, regardless of whether C3 was purified from samples from controls or patients with type 1 diabetes. Moreover, affimer A6 was capable of reducing plasma clot lysis in samples from both healthy controls and patients with diabetes. We and others have shown that changes in clot lysis by $6-18 \%$ are clinically significant $t^{2426}$ and therefore the observed $7-$ $11 \%$ reduction in clot lysis by affimer A6 is likely to be clinically meaningful. Although speculative, this targeted effect on clot lysis is unlikely to increase bleeding risk significantly, making this approach clinically promising.

The predicted binding sites of both loops of affimer A6 were in close proximity within the $\beta$ chain of fibrinogen and close to the region of fibrinogen that interacts with C3 as determined by microarray screening. Considering the data together, it appears that the $\mathrm{N}$ terminus of the $\beta$ chain of fibrinogen is a binding site for complement C3,
A

\begin{tabular}{cc}
\hline Sample & Lysine residue \\
\hline 1 & $685,1203,1209$, \\
2 & $685,1203,1209$, \\
3 & $685,1203,1209,1526$ \\
4 & $502,1203,1209$ \\
5 & 502,1139 \\
6 & 502,1139 \\
\hline
\end{tabular}

B

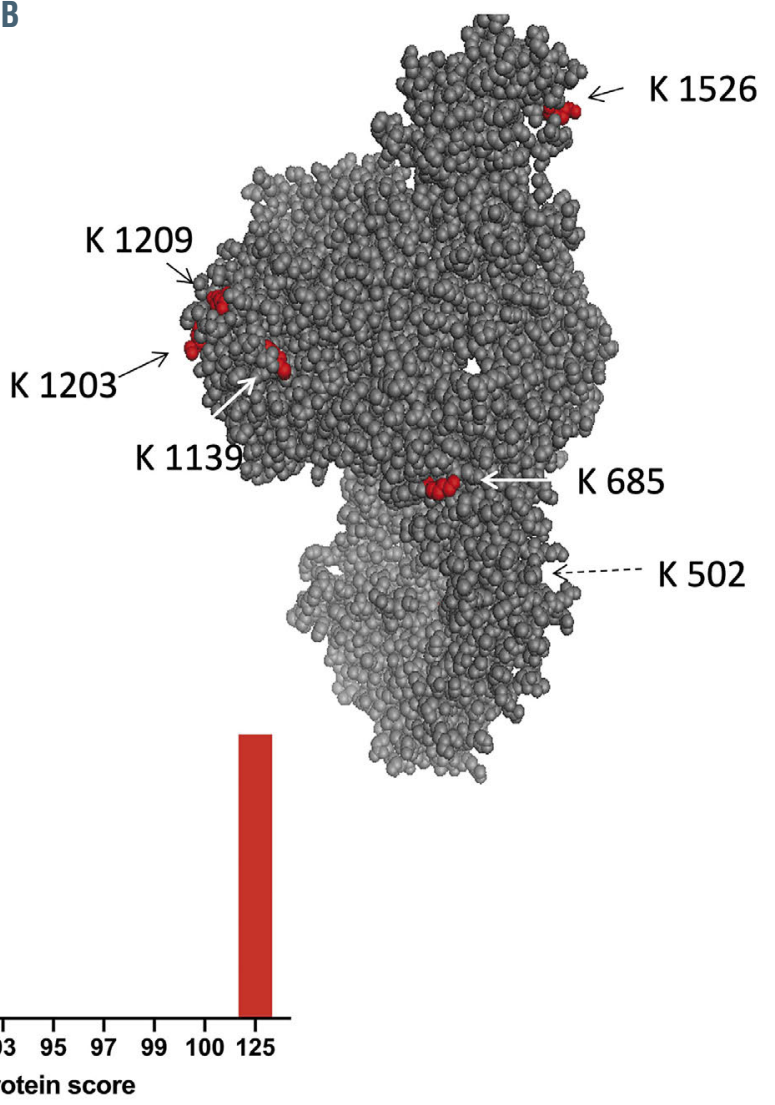

C

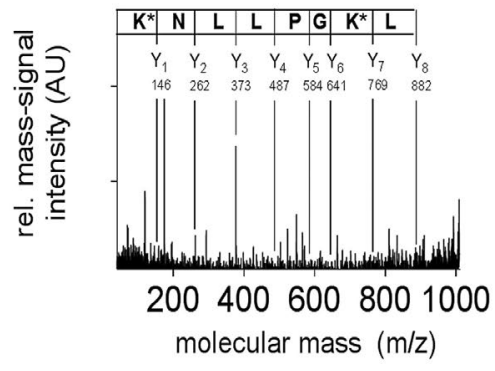

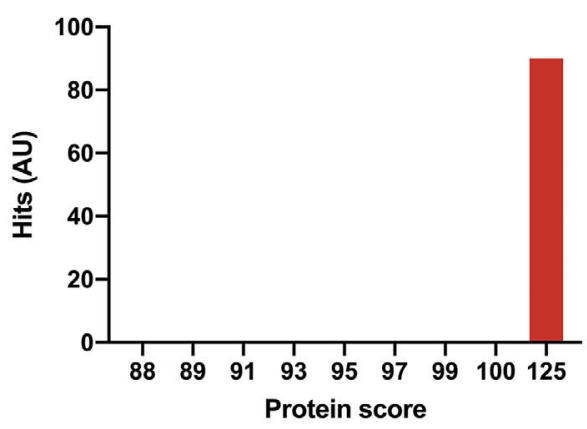

Figure 5. In-vivo glycation of C3 and lysine residues involved. Six individual C3 samples from patients with type 1 diabetes and healthy controls were analyzed by matrix-assisted laser desorption/ionization mass-spectrometry (MALDI-MS) for post-translational glycation. (A) Six lysine residues showed additional glycation in samples from the patients with diabetes. (B) The spatial position of glycated residues within C3 are shown. (C) Detailed analysis of the tandem mass spectrometry fragment from C3 with the molecular mass of 1,206 Daltons and the interpretation by the Mascot search engine is shown together with a protein score of 125 for post-translational glycation modification. AU: arbitrary units. 
which may be important for the development of agents that improve the efficiency of fibrinolysis, particularly in patients with high vascular risk. These findings open a new avenue for the identification of therapeutic targets to modulate the hypofibrinolytic environment in highrisk patients, which may have future clinical implications.

In conclusion, our work shows that C3 represents a credible therapeutic target to reduce thrombosis risk. The $\beta$ chain of fibrinogen represents an interaction site with C3, and modulation of this interaction can abolish C3induced prolongation of clot lysis in a specific manner. This targeted approach has the potential for use in the development of novel therapeutic agents for the reduction of thrombotic vascular occlusion.

Future work should concentrate on investigating the in vivo role of affimer A6 in limiting vascular occlusion, using animal models of thrombosis. Additionally, our data suggest that a specific site of the $\beta$ chain of fibrinogen, which may be amenable to small molecule intervention, represents a possible new therapeutic target to improve fibrinolysis and reduce thrombosis in high-risk conditions.

\section{Disclosures}

No conflicts of interest to disclose.

\section{Contributions}

$R J K$ and KS performed the research, analyzed data and wrote the manuscript. CT, VJ and KS performed the research, analyzed data and revised the manuscript. VJ and AT researched data and revised the manuscript. SP, MJM and RFS revised the manuscript. CWGF and DT designed experiments and revised the manuscript. RAA conceived the study, designed experiments, analyzed data and wrote the manuscript.

\section{Funding}

RJK was funded by a PhD Fellowship from the Sir Jules Thorn Charitable Trust. KS is supported by the German Research Foundation (DFG) [SFB/TRR219 C-07; HE 5666/1-2 to KS (née Hess)] and by a grant from the Interdisciplinary Center for Clinical Research within the faculty of Medicine at the RWTH Aachen University (K7-2). VJ was supported by the German Research Foundation (DFG)(SFB/TRR219 S-03, C-04, M-05, IHST 948/4S-1 FU6.6). We also wish to thank Diabetes UK for funding part of this work, the National Institute of Health Research and British Heart Foundation for constant support, along with help from the Biomedical Health Research Centre for the BioScreening Technology Group and the Stratified Medicine Hub at the University of Leeds who funded the initial NABP screens. We would also like to thank AVACTA Life Sciences for their support.

\section{References}

1. Lisman T. Decreased plasma fibrinolytic potential as a risk for venous and arterial thrombosis. Semin Thromb Hemost. 2017;43(2):178-184

2. Kearney K, Tomlinson D, Smith K, Ajjan R. Hypofibrinolysis in diabetes: a therapeutic target for the reduction of cardiovascular risk. Cardiovasc Diabetol. 2017;16(1):34.

3. Undas A. Fibrin clot properties and their modulation in thrombotic disorders. Thromb Haemost. 2014;112(1):32-42.

4. Sumaya W, Wallentin L, James SK, et al. Fibrin clot properties independently predict adverse clinical outcome following acute coronary syndrome: a PLATO substudy. Eur Heart J. 2018;39(13):1078-1085

5. Eikelboom JW, Connolly SJ, Bosch J, et al. Rivaroxaban with or without aspirin in stable cardiovascular disease. $N$ Engl J Med. 2017;377(14):1319-1330.

6. Hertle E, Stehouwer CD, van Greevenbroek $\mathrm{MM}$. The complement system in human cardiometabolic disease. Mol Immunol. 2014;61(2):135-148.

7. Hess K, Alzahrani SH, Mathai M, et al. A novel mechanism for hypofibrinolysis in diabetes: the role of complement C3. Diabetologia. 2012;55(4):1103-1113.

8. Howes JM, Richardson VR, Smith KA, et al. Complement C3 is a novel plasma clot component with anti-fibrinolytic properties. Diab Vasc Dis Res. 2012;9(3):216-225.

9. Hess $\mathrm{K}$, Alzahrani $\mathrm{SH}$, Price JF, et al. Hypofibrinolysis in type 2 diabetes: the role of the inflammatory pathway and comple- ment C3. Diabetologia. 2014;57(8):17371741.

10. Kearney KJ, Pechlivani N, King R, et al. Affimer proteins as a tool to modulate fibrinolysis, stabilize the blood clot, and reduce bleeding complications. Blood. 2019;133 (11):1233-1244.

11. Tiede C, Bedford R, Heseltine SI, et al. Affimer proteins are versatile and renewable affinity reagents. Elife. 2017;6:e24903.

12. Tiede C, Tang AA, Deacon SE, et al. Adhiron a stable and versatile peptide display scaffold for molecular recognition applications. Protein Eng Des Sel. 2014;27(5):145-155.

13. Carter AM, Cymbalista CM, Spector TD, Grant PJ. Heritability of clot formation, morphology, and lysis: the EuroCLOT study. Arterioscler Thromb Vasc Biol. 2007;27(12): 2783-2789.

14. Harris R, Olson AJ, Goodsell DS. Automated prediction of ligand-binding sites in proteins. Proteins. 2008;70(4):1506-1517.

15. Trabuco LG, Lise S, Petsalaki E, Russell RB. PepSite: prediction of peptide-binding sites from protein surfaces. Nucleic Acids Res. 2012;40(Web Server issue):W423-W427.

16. Rueth $M$, Lemke $H D$, Preisinger $C$, et al. Guanidinylations of albumin decreased binding capacity of hydrophobic metabolites. Acta Physiol (Oxf). 2015;215(1):13-23.

17. Kimmel J. Guanidation of proteins. Meth Enzymol. 1967:11:584-589.

18. Kollman JM, Pandi L, Sawaya MR, Riley M, Doolittle RF. Crystal structure of human fibrinogen. Biochemistry. 2009;48(18):38773886

19. Balakumar P, Maung U, Jagadeesh G. Prevalence and prevention of cardiovascular disease and diabetes mellitus. Pharmacol Res. 2016;113(Pt A):600-609.

20. Pieters M, van Zyl DG, Rheeder P, et al. Glycation of fibrinogen in uncontrolled diabetic patients and the effects of glycaemic control on fibrinogen glycation. Thromb Res. 2007;120(3):439-446.

21. Pieters M, Covic N, Loots DT, et al. The effect of glycaemic control on fibrin network structure of type 2 diabetic subjects. Thromb Haemost. 2006;96(5):623-629.

22. Ajjan RA, Gameln T, Standeven KF, et al Diabetes is associated with post-translationa modifications in plasminogen resulting in reduced plasmin generation and enzyme specific activity. Blood. 2013;122(1):134-142.

23. Smith KA, Pease RJ, Avery CA, et al. The activation peptide cleft exposed by thrombin cleavage of FXIII-A(2) contains a recognition site for the fibrinogen alpha chain. Blood. 2013;121(11):2117-2126.

24. Neergaard-Petersen S, Hvas AM, Kristensen $\mathrm{SD}$, et al. The influence of type 2 diabetes on fibrin clot properties in patients with coronary artery disease. Thromb Haemost. 2014:112(6):1142-1150.

25. Undas A, Wiek I, Stepien E, Zmudka K, Tracz W. Hyperglycemia is associated with enhanced thrombin formation, platelet activation, and fibrin clot resistance to lysis in patients with acute coronary syndrome. Diabetes Care. 2008;31(8):1590-1595.

26. Alzahrani SH, Hess K, Price JF, et al Gender-specific alterations in fibrin structure function in type 2 diabetes: associations with cardiometabolic and vascular markers. J Clin Endocrinol Metab. 2012;97 (12):E2282-E2287. 